\title{
Histopathological Studies on Intra Epididymal Zinc Arginine Administration in Pre-Pubertal Dogs
}

\section{Rajan Chaudhary ${ }^{1}$, Arun Kumar Sharma ${ }^{2}$, Umed Singh Mehra ${ }^{1 *}$, Ravi Dutt ${ }^{2}$, Pankaj Kumar ${ }^{3}$ and Rajendra Yadav ${ }^{4}$}

${ }^{1}$ Department of Animal Husbandry and Dairying, ${ }^{2}$ Department of Veterinary Gynaecology and Obstetrics, ${ }^{3}$ Department of Veterinary Public Health and Epidemiology, LUVAS, Hisar, Haryana-125004, India

${ }^{4}$ Regional Referral Veterinary Diagnostic and Extension Centre (RVDEC), Mahendergarh, India

*Corresponding author

\section{A B S T R A C T}

\section{Keywords}

Cauda epididymis, Contraceptive methods, Dogs, Pre-pubertal, Zinc arginine

\section{Article Info}

Accepted:

07 April 2018

Available Online:

10 May 2018
Stray dog bite is one of the public health problem in the world and there is a great need of envisaging irreversible contraceptive methods in dogs. Therefore, the present study was conducted to evaluate the effects of intra epididymal zinc arginine administration in prepubertal dogs. Eighteen pre-pubertal stray community dogs weighing 6-8 kg were administered with zinc arginine solution@ $0.2 \mathrm{ml}$ per each cauda epididymis percutaneously using 26 gauge, 1/2 inch needle. On day 10, 20, 30, 40, 50, 60, 70, 80 and 90 post injection two separate animals were subjected to surgical removal of testis and epididymis for histopathological studies. Similarly, one untreated control was operated on day 10 and second on day 90 of experiment. From the present study it is concluded that single intra-epididymal injection of zinc arginine causes complete necrosis of the cauda epididymal tubules and irreversible loss of fertility in dogs.

\section{Introduction}

Public in almost every metropolitan city, town and even villages is facing unprovoked dog bites from stray dogs roaming free in their localities. Similarly, though the exact human and domestic animal mortality due to rabies is not known but the seriousness of the problem can be estimated from the fact that every year about 300-500 cases are admitted in the only infectious disease hospital in Delhi alone. Dogs are responsible for $>90 \%$ of the estimated 55,000 human deaths and for the millions of people that each year receive postexposure prophylaxis following a bite (Knobel et al., 2005).

Controlling reproduction in stray dogs remains critically important, since this strategy is proactive rather than reactive. After excluding the possibility of euthanasia, population control either by surgical or non-surgical method is the only choice left to control the ever- increasing stray dog population and thereby reduce the incidence of dog bites and ultimately rabies. However, the issue of how 
best to reduce the number of stray dogs is complex and multifaceted in a country like India and involves political, ethical, environmental, financial as well as technical considerations. Since males are always potentially capable of impregnating a number of receptive females, so sterilizing number of males could conceivably decrease the number of pregnant female to the point of reversing population growth. It is imperative that the technique should be simple, non- surgical, least invasive, inexpensive, permanent, reliable, painless and simple for field functionaries and require no post- treatment hospitalization and care. Non- surgical method would facilitate sterilization of large population of stray dogs in shortest possible time because it will not require commitment of technical expertise, equipment and expenses.

Various chemicals that have been used for sterilization of male dogs are $10 \%$ silver nitrate, 3.6\% formaldehyde (Freeman and Coffey,1973); methalibure, dexamethasone, metopiron, alpha chlorohydrin, danazole (Dixit et al., 1975); chlorohexidine gluconate, (Pineda et al., 1977; Brijlal, 2002); calcium chloride (Koger, 1978; Dutta et al., 1993), cadmium chloride (Murty and Sastry, 1978), zinc arginine (Fahim et al., 1993; Tepsumethanon et al., 2005), and 70\% Glycerol (Immegart and Treefall, 2000).

However out of all these chemicals, chlorohexidine gluconate and zinc arginine have proved to be promising. The later has been approved by FDA of USA for sterilization of young pups and is being marketed in USA with the trade name of Neutersol. But no work has been done on this aspect in India. Keeping these facts and findings in mind, the present study was planned to study the effects of intra epididymal zinc arginine administration in pre-pubertal dogs.

\section{Materials and Methods}

The present study was conducted on 22 apparently healthy pre-pubertal stray community dogs weighing 6-8 kilograms. Dogs were caught from the local population and were kept under observation for one week prior to the start of the experiment. All the dogs were housed in individual cages. For experimental studies, permission from Institutional Animal Ethics Committee was taken. For eliminating the diseased dogs from the healthy ones standard haematological, parasitological and biochemical examination of blood, faeces and urine were conducted. Gross examination of the scrotum, penis and epididymis was performed. Testicles of each dog were examined for smoothness, firmness, tone, size and adhesion if any. Selected animals were weighted, treated for external and internal parasites and immunized against rabies. Shaving of the scrotal area was performed and surgical spirit solution was applied to disinfect the area.

Before giving intra epididymal injection dogs were anaesthetized with an intra-muscular injection of Ketamine $\mathrm{HCl}(2.4 \mathrm{mg} / \mathrm{kg} \mathrm{B}$.Wt.) and Xylazine $(10 \mathrm{mg} / \mathrm{kg}$ B.Wt.). The Cauda epididymis was located by tightening the skin of the scrotum and by pushing the testis in the scrotal sac. Zinc arginine solution@ $0.2 \mathrm{ml}$ per each cauda epididymis was injected percutaneously using 26 gauge, $1 / 2$ inch needle in 18 dogs. This zinc arginine solution was a 0.4 $M$ solution of zinc gluconate neutralized by equimolar solution of L-arginine, achieving a final concentration of $100 \mathrm{mg} / \mathrm{ml}$ of zinc arginine of solution. The $\mathrm{pH}$ of the solution was adjusted to 7 using N/10 HCl. Four dogs kept as untreated control. Two out of four dogs completed the experiment. Animals were examined regularly upto $96 \mathrm{hrs}$ for inflammation, swelling, oedema, pain etc. in the scrotum and cauda epididymis after the injection of zinc arginine solution. 
Testes along with epididymis were surgically removed from two separate dogs on day 10 , $20,30,40,50,60,70,80$ and 90 of zinc arginine injection and complete post-operative care was given to all the operated animals. Testes and epididymis from control first animal were removed on day 10 and from second animal on day 90 of experiment. Tissues were dehydrated in methanol and cleared in Xylene, then paraffin blocks were prepared. Sections were cut at 5-7 $\mu$ and tissues were stained with Harris-Haematoxylin and Eosin (H\&E). Epididymal tissue sections were examined for microscopic changes in their shape, size, lining of the epithelium of the tubules, infiltrations of different cell types and granulomatous changes and compared with epididymal histology of control dogs. Similarly, seminiferous tubules were examined with respect to type of cells using Haematoxylin and Eosin stained slides. The cycle of the seminiferous epithelium was divided into eight stages on the basis of the morphology of the germinal cells, their relative position within the seminiferous tubules and cellular associations. The criteria described by Foote et al., (1972) were used for scoring the stages of the seminiferous epithelial cycle using haematoxylin and eosin stained slides.

\section{Results and Discussion}

The following histological changes in the epididymis at different time interval after zinc arginine administration were observed:

\section{Day 10 post injection}

\section{Cauda}

Inter-tubular tissue became thickened due to proliferation of fibrous connective tissue. There was infiltration of leukocytes (Fig. 1). Tubular epithelium showed degenerative changes. Stereocilia present in the healthy tubules were totally absent. The height of the columnar cells of the epithelial lining tubules decreased considerably. There was a flattening of the cells with little cytoplasm.

\section{Corpus}

There was proliferation of fibrous connective tissue and infiltration of leucocytes in the inter-tubular areas. Degenerative changes in the walls of the tubules were seen. Cilia were damaged and height of the cells of epithelium lining the walls of the tubules decreased. Degenerative changes in the corpus were of low intensity as compared to cauda (Fig. 2).

\section{Caput}

Proliferation of fibrous connective tissue in the inter-tubular areas and infiltration of leucocytes was noticed. Degenerative changes were noticed in the walls of the tubules. Cilia were completely absent from the tubules. Height of the cells of the epithelium lining tubular walls was very less as compared to the height of cells in healthy tubules. The degenerative changes in the caput were of low intensity as compared to changes in corpus or cauda (Fig. 3).

\section{Day 20 post injection}

\section{Cauda}

There was extensive fibrous tissue proliferation in the inter-tubular areas. Hyaline degeneration in most of the areas (Fig. 4) and infiltration of macrophages was observed. Severe necrotic changes were observed in all most all the tubules.

\section{Corpus}

The proliferation of the fibrous connective tissue increased further in the inter-tubular areas. Hyaline degenerative changes in the 
most of the areas occurred. The tubular walls were without any cilia. The cells of the epithelial lining in epididymal tubules were flattened with little cytoplasm. The changes in the tubules of the corpus were of very low intensity (Fig. 5) as compared to changes in the cauda.

\section{Caput}

Proliferation of the connective tissue in the inter-tubular area could be seen. The epithelium of the tubules in caput was almost normal. At higher magnification, cilia present in the tubules could be appreciated (Fig. 6). The size of the tubules decreased as compared to healthy tubules of the caput.

\section{Day 30 post injection}

\section{Cauda}

Nearly complete degeneration of the epididymal tubules took place. Almost whole of the area was covered by fibrous connective tissue. A few tubules undergoing degenerative changes could still be seen. Most of the tubules had lost their structural details (Fig. 7).

\section{Corpus}

The picture here was similar to that seen on day 20 post injection changes in corpus. Size of the tubules was smaller and cilia were absent. Inter-tubular connective tissue was more as compared to healthy tubules (Fig. 8).

\section{Caput}

Inter-tubular fibrous connective tissue proliferation was present but less marked as compared to corpus. Epithelium lining the tubules was almost normal (Fig. 9). Cilia present in the tubules could be very well appreciated. The size of the tubules was smaller as compared to healthy tubules.

\section{Day 40 post injection}

\section{Cauda}

Complete degeneration of the tubules had taken place. No tubule could be recognized in the field. The whole of the area was lined by fibrous connective tissue (Fig. 10). The cauda epididymis had completely lost its structural details and hyaline degenerative changes occurred in the connective tissue.

\section{Corpus}

The tubules and inter-tubular area were similar to that seen in the case of corpus day 30 post injection changes. The cells of the epithelium lining the tubules were flattened type instead of healthy ciliated columnar cells. Complete absence of cilia in the tubules of corpus (Fig. 11).

\section{Caput}

Changes similar to that seen in the caput day 30 post injection. Almost healthy tubules lined by epithelium having columnar type of ciliated cells were observed (Fig. 12).

\section{Control dogs}

\section{Epididymis}

All the tubules in cauda (Fig. 13), corpus (Fig. 14a) and caput (Fig. 14b) were healthy and lined by pseudo-stratified epithelium having columnar type of ciliated cells on day 10 of experiment. The seminiferous tubules of testes were also healthy on day 10 of experiment. The tubules were lined with several layers of different generations of germ cells (Fig. 15).

\section{Day 50 post injection}

In cauda all the tubules had been replaced by connective tissue. 
Fig.1 Cauda $10^{\text {th }}$ day post-injection (X400)

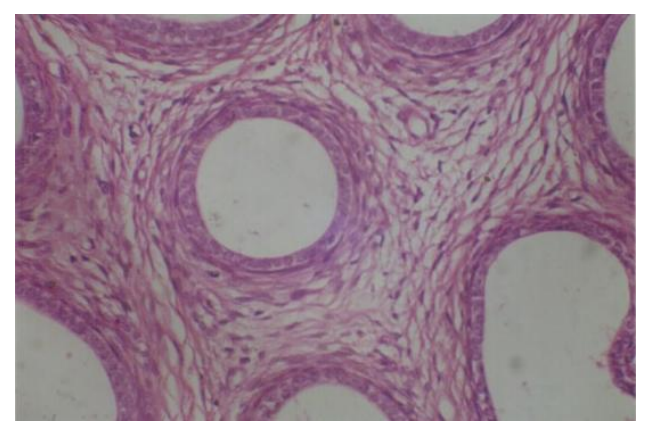

Fig. 2 Corpus $10^{\text {th }}$ day post-injection (X400)

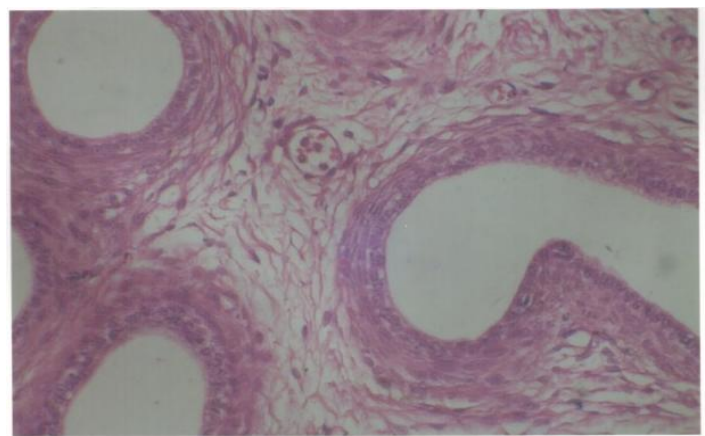

Fig.3 Caput $10^{\text {th }}$ day post-injection (X400)

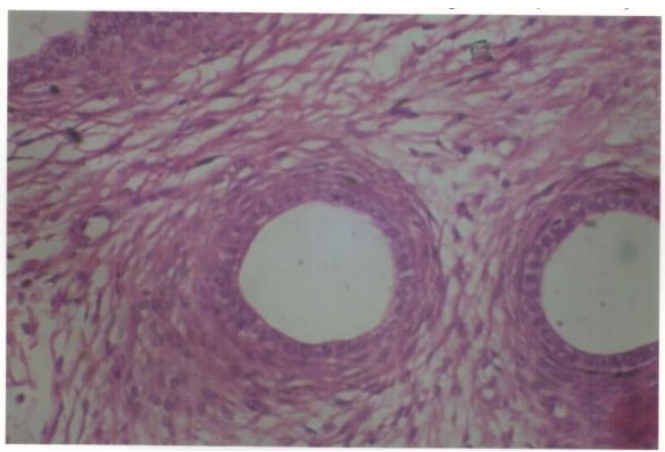

Fig.4 Cauda $20^{\text {th }}$ day post-injection (X400)

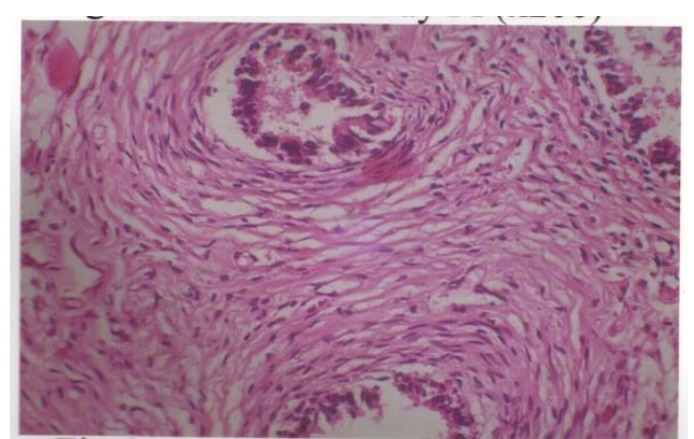


Fig.5 Corpus $20^{\text {th }}$ day post-injection (X400)

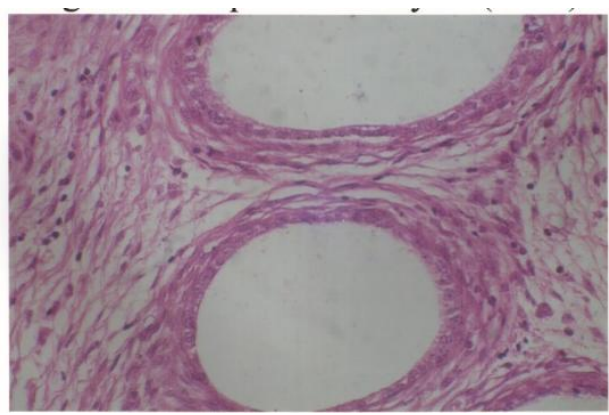

Fig.6 Caput $20^{\text {th }}$ day post-injection (X400)

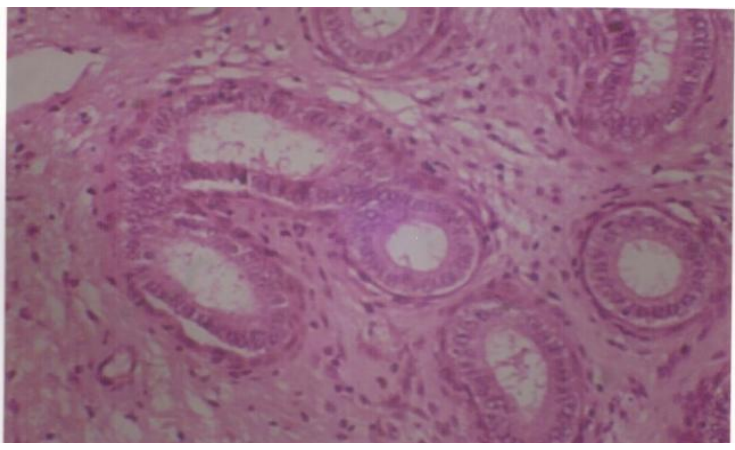

Fig.7 Cauda $30^{\text {th }}$ day post-injection (X400)

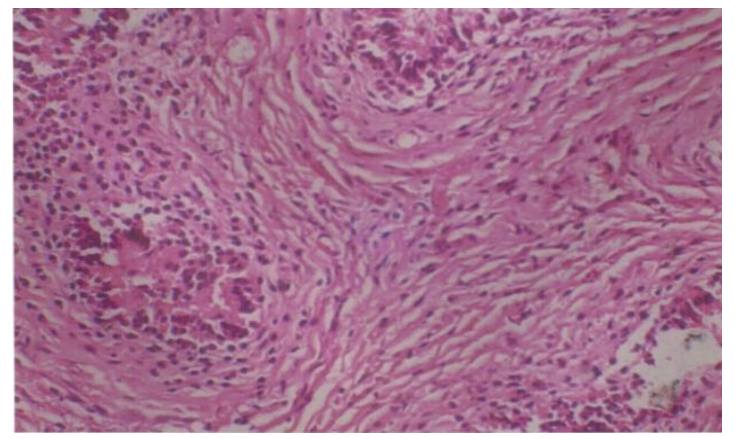

Fig.8 Corpus $30^{\text {th }}$ day post-injection (X400)

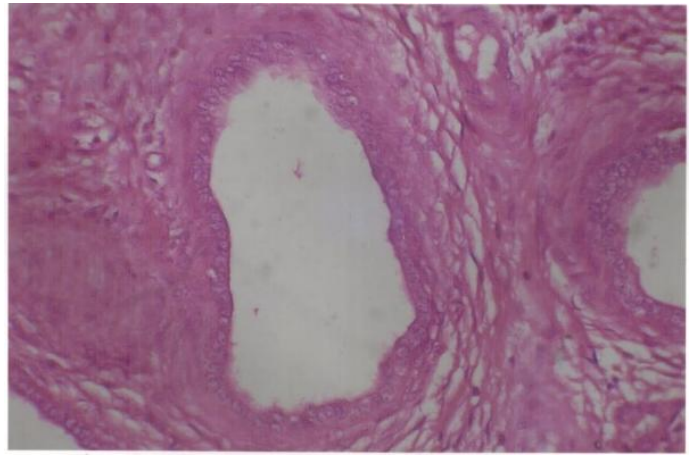


Fig.9 Caput $30^{\text {th }}$ day post-injection (X400)

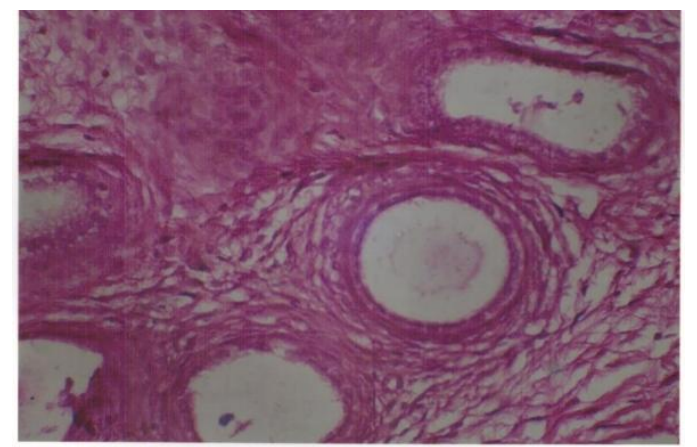

Fig.10 Cauda $40^{\text {th }}$ day post-injection $(\mathrm{X} 400)$

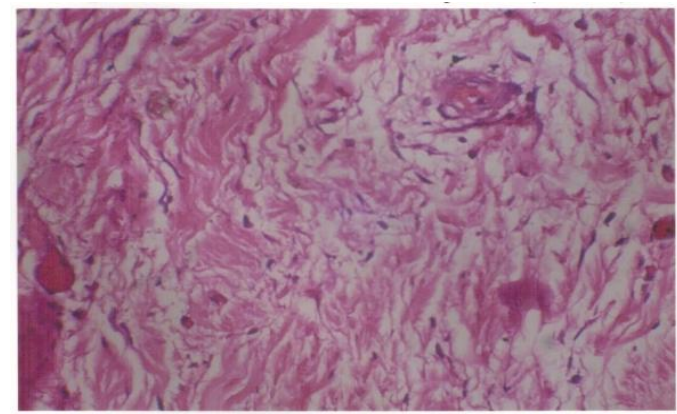

Fig.11 Corpus $40^{\text {th }}$ day post-injection (X400)

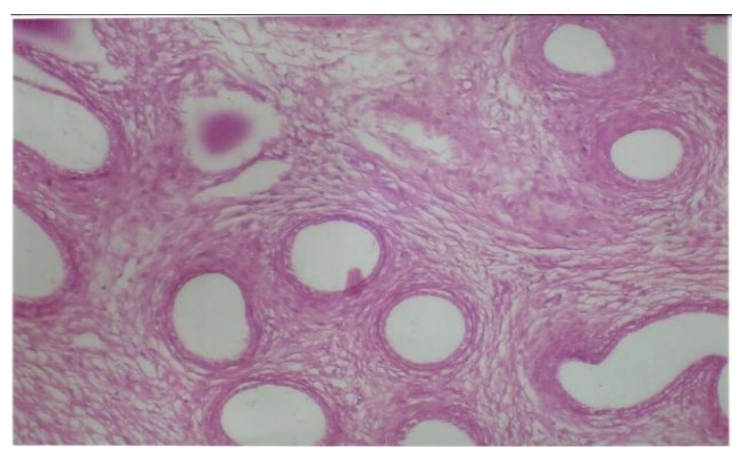

Fig.12 Caput $40^{\text {th }}$ day post-injection $(\mathrm{X} 400)$

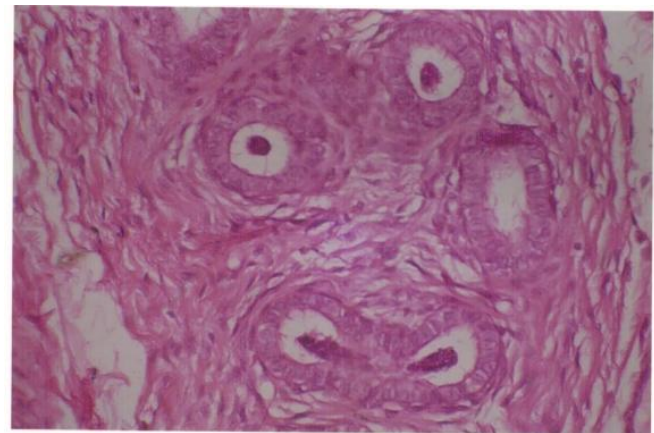


Fig.13 Control cauda (X 400) Fig.14a Control corpus (X 400) Fig.14b Control caput (X 400)
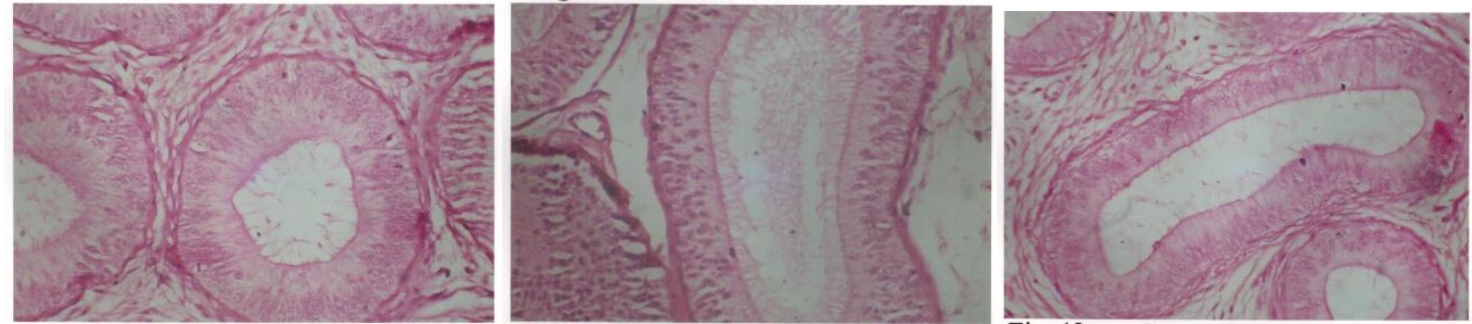

Fig.15 Control testis (X 400)

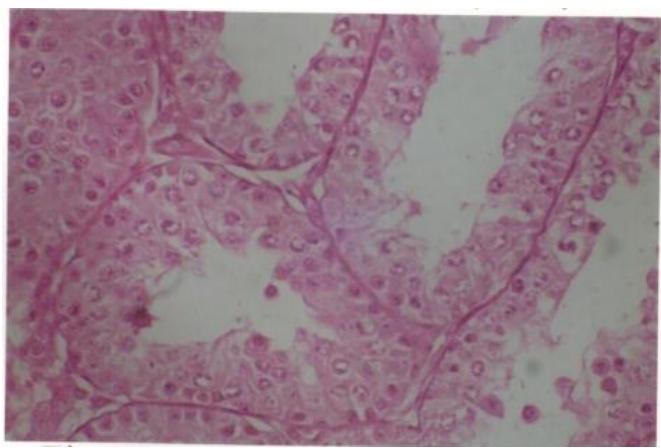

Fig.16 Caput $50^{\text {th }}$ day post-injection (X400)

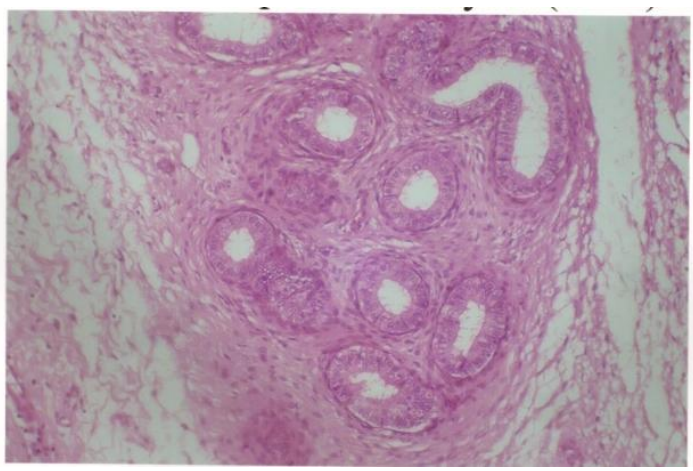

Fig.17 Corpus $70^{\text {th }}$ day post-injection (X400)

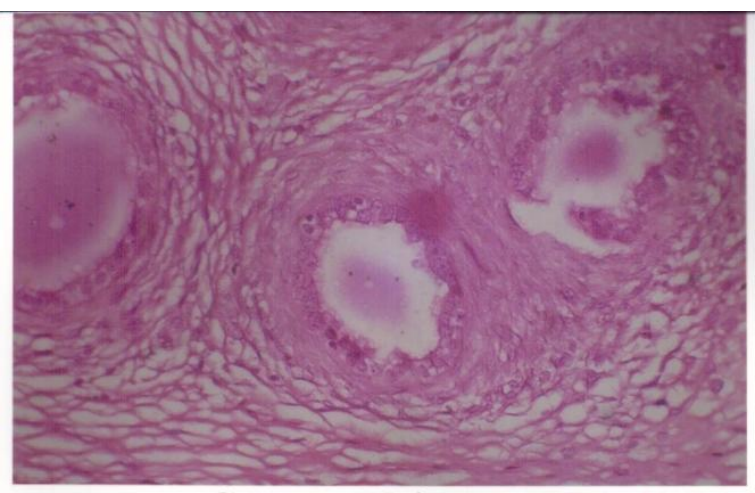


Fig.18 Cauda $80^{\text {th }}$ day post-injection (X400)

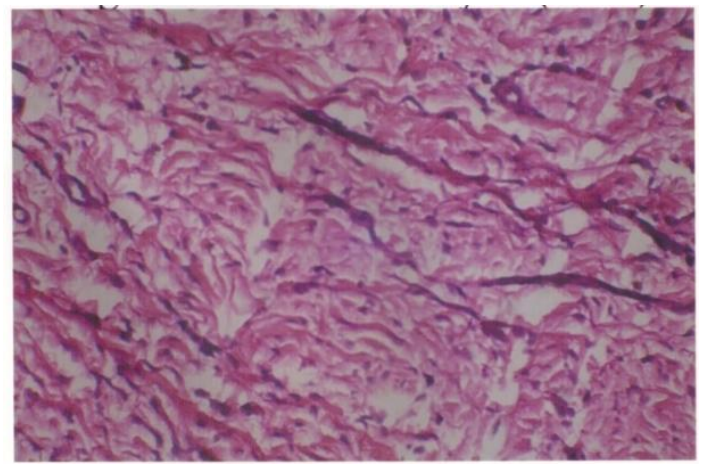

Fig.19 Corpus $90^{\text {th }}$ day post-injection (X400)

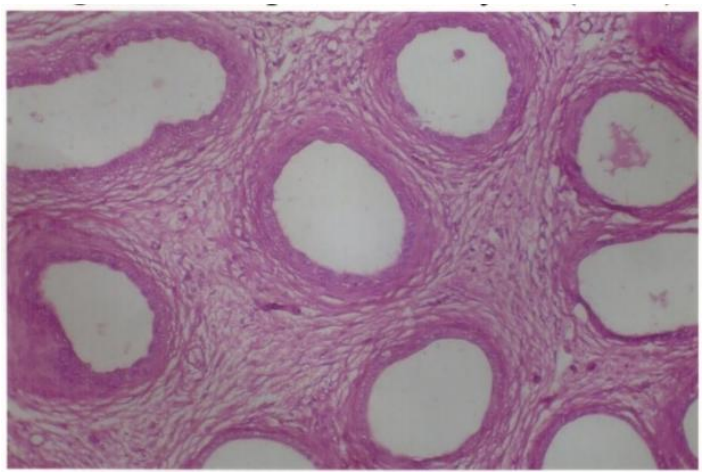

Picture was similar to that seen in the case of cauda day 40 post injection. In corpus, no further changes in the tubular wall or inter tubular area were observed.

Picture was similar to that seen in the case of corpus day 40 injection. In caput, almost healthy tubules though smaller in size could be seen. Tubules were lined by tall columnar cells having abundance of cytoplasm and stereocilia (Fig. 16).

\section{Day 60 post injection}

In cauda, changes similar to that seen in the case of cauda by 40 post injection. Similarly, in corpus the picture very much similar to that seen in the corpus day 40 post injection.

In caput, no deviation from the finding of caput day 50 post injection.

\section{Day 70 post injection}

In cauda, picture of changes similar to that seen in the case of cauda day 40 or 50 post injection. In corpus, the size of the tubules was smaller in comparison to healthy tubules and the cilia were absent. Cells lining the tubules were flattened instead of healthy tall columnar cells (Fig. 17). In caput, ciliated columnar type of cells was lining the healthy tubules. Lumen of these tubules was empty and compared to healthy tubules, these tubules were smaller in size.

\section{Day 80 post injection}

In cauda, only connective tissue could be seen in the field (Fig. 18). In corpus, changes were similar to that seen in the case of corpus day 70 post injection. There was increased intertubular connective tissue as compared to 
healthy corpus epididymis. Few healthy tubules were seen in caput but their lumen was empty.

\section{Day 90 post injection}

In cauda, the findings were similar to the earlier ones. No tubules only connective tissue was present. Tubules were having empty lumen in corpus and the connective tissue proliferation in the inter-tubular areas could be seen (Fig. 19). Thickness of the walls of the tubules was less and cilia were absent.

The tubules were smaller in size as compared to healthy tubules in caput.

\section{Control dogs}

In epididymis all the tubules in cauda, corpus and caput and seminiferous tubules of testes were healthy on 90 of the experiment. These tubules were lined by pseudo stratified epithelium having columnar type of ciliated cells.

In the current study, on day 30 post injection, a complete degeneration of epididymal tubules was observed and the whole area was covered by fibrous tissue and most of the tubules had lost their structural details. Fahim et al., (1993) conducted the histopathology of epididymis one year after the injection of zinc arginine in adult dogs and observed findings similar to current investigation. It is presumed that these changes might have been taken place a long time ago. Pineda et al., (1977) while conducting similar type of studies with chlorohexidine gluconate in pre-pubertal and adult male dogs noted similar changes at day 358 post injection. Pineda and Dooley (1984) reported similar changes in toms at day 252 post injection. In the above experiments, the tissues for histopathology were taken after a long period post injection. So, it was difficult to ascertain the time at which these changes would have taken place. Similar type of necrotic changes in the tubular walls and proliferation of fibrous tissue in the intertubular areas were observed by Brijlal (2002) and Singh (2004) in cow bulls at day and 21 post injection using chlorohexidine gluconate. These workers reported the formation of sperm granulomas in the cauda epididymis. But, in the present study there were no granulomas as the dogs were pre-pubertal. Fahim et al., (1993) while conducting similar experiments with zinc arginine in adult dogs also did not observed any granulomas. While many other workers reported formation of sperm granulomas (Pineda et al., 1977; Pearson et al., 1980 and Pineda and Dolley, 1984).

The concentration at which zinc arginine was injected into the cauda epididymis might be toxic to the cells and Tissues of cauda epididymis resulting in infiltration of leukocytes. This inflammatory reaction later on becomes chronic which is evident from fibrous tissue proliferations of the intertubular areas. The necrotic changes observed in the present investigation in cauda epididymis were severe than the changes reported by other workers at this stage and thereafter. This may be due to fact that young growing cells of the cauda epididymides are more sensitive to toxins or toxic changes in the environment as compared to fully grown adult cells. In current investigation, at day 40 post injection, a complete degeneration of all the tubules of cauda epididymis had taken place, whole of the area was lined by fibrous connective tissue and the cauda epididymis had lost its structural details. Similar changes were reported by Bierschwal and Ebert (1961) on day 12 post injection. They reported complete loss of structural details of cauda epididymis after 'Dondren' injection. No other worker reported complete degeneration of epididymal tubules. This may be due to the 
fact that majority of research work had been performed in the adult animals. From day 40 to day 90 post injection, no further structural changes were observed in cauda, corpus or caput epididymis. While Tepsumethanon et al., (2005) documented histopathological finding that virtually complete with fibrosis of seminiferous tubules and Leydig cells occurs on days 60 and 75 post injection. Vannucchi et al., (2015) reported that intratesticular injection of zinc gluconate associated with DMSO, two months after resulted in reduction of testicular volume and changes of testicular echotexture including degeneration, fibrosis, and calcification of testicular parenchyma on histologic examination.

In this study, the seminiferous tubules only upto $4^{\text {th }}$ stage of seminiferous epithelium cell cycle were present. The tubules were lined with several layers of different generation of germ cells. Histological examination of testicular tissue revealed normal germinal epithelium in all the treated dogs. Spermatid was not seen in any of the seminiferous tubules as the dogs were pre-pubertal. All the cellular elements that is spermatogonia (Type A, type B and intermediate) and primary spermatocytes were normal and did not reveal any qualitative changes. The seminiferous tubules only upto $4^{\text {th }}$ stage of seminiferous epithelial cell cycle were present. The tubules were lined with several layers of different generations of germ cells. Spermatogenesis was observed to be normal qualitatively and quantitatively in all treated dogs. Similar finding was reported by Fahim et al., (1993) in adult dogs who observed no changes in cellular structure of testes of treated dogs, one year after intra epididymal injection of zinc arginine. However, Vannucchi et al., (2015) contradicted and concluded that impairment of sperm quality-related variables was observed 15 days after the first intra-testicular administration of zinc gluconate and $\mathrm{di}$ methyl sulfoxide (i.e., decrease in sperm count and sperm motility and an increase in major sperm defects and by this a decrease in the total number of morphologically normal and motile sperm).

The size of the cauda epididymis was very small as the dogs used in the present study were pre-pubertal. Also, there were chances of leakage of solution from cauda epididymis or faulty deposition of solution in the scrotum. Therefore, it seems likely that as the zinc arginine solution comes in the contact with parietal tunica vaginalis of scrotum, it causes irritation and local tissue reaction which leads to pain, biting and licking, scrotal swelling etc. and later on adhesions are formed. Injection of zinc gluconate/arginine effectively reduces testosterone levels achieving sterility while maintaining normal body function (Min Wang et al., 2013). Cavalieri et al., (2015) concluded that a single, intra-testicular administration of either $57.75 \mathrm{mg}$ or $71.75 \mathrm{mg}$ of zinc arginine solution was able to reduce circulating concentrations of testosterone without significantly affecting live weights or carcass weights. Thus, low cost, ease of use, and cultural acceptance of a castration technique that does not require removal of the testes make zinc arginine a valuable option for large-scale use in dogs, particularly in remote locations lacking sophisticated clinical facilities or skilled surgeons.

The present investigation was carried out on twenty-two pre-pubertal stray community dogs, of which eighteen dogs were injected $0.2 \mathrm{ml}$ of $0.4 \mathrm{M}$ solution of zinc arginine into each cauda epididymis to study the effect of zinc arginine on epididymis histology and spermatogenesis at different intervals of time. On days 20 and 30 post injection the changes observed in the cauda epididymis are extensive fibrous tissue proliferation, infiltration of macrophages, hyaline degeneration in most of the areas, complete 
degeneration of the tubules leading to complete loss of structural details of the tubules. Later on, cauda epididymal tubules are replaced by fibrous connective tissue and loss its structural details. The changes in the cauda epididymis appeared to be irreversible in treated dogs. While in corpus and caput, healthy tubules were present having only mild degenerative changes. The size of these tubules was smaller than normal tubules. The germinal epithelium of seminiferous tubules in treated dogs was normal and had active spermatogenesis. No qualitative of quantitative changes were observed in seminiferous tubules. So from the present study it is concluded that single intraepididymal injection of zinc arginine causes complete necrosis of the cauda epididymal tubules in pre- pubertal dogs. Therefore, it is concluded that single intra-epididymal injection of zinc arginine can cause an irreversible loss of fertility in dogs.

\section{References}

Bierschwal, C.J. and Ebert, E.P. (1961). Clinical application of a scleratherapeutic agent. Vet. Med. 56: 323-332.

Brijlal (2002). Effects of chlorohexidine gluconate on epididymis and spermatogenesis in dogs. M.V.Sc Thesis, CCSHAU, Hisar (Haryana), India.

Cavalieri, J., Wang, M. and Johnson, L. (2015). Chemical sterilisation of Bos indicus bull calves following intratesticular injection of zinc acetate: Effects on growth and concentrations of testosterone. Anim. Repro. Sci., 159: 163-171.

Dixit, V.B., Lohiya, N.K., Arya, M. and Agrawal, M. (1975). Chemical sterilization of male dogs after a single intratesticular injection of "Danazole". Folia. Biol. (Krakow). 23(3): 505-510.
Dutta, A.B., Maji, A.K. and Samanta, P.K. (1993). Comparative evaluation of calcium chloride, cadmium chloride and "Talsur" in chemo-sterlization of male dogs. $16^{\text {th }}$ annual congress ISVS and National Symposium, held on Jan. 2022 at Parbhani, Maharastra (India).

Fahim, M.S., Wang, M., Suteu, M.P., Fahim, Z. and Youngquist, R.S. (1993). Sterlization of dogs with intra epididymal injection of Zinc arginine. Contraception stoneham. 47(1): 107122.

Foote, R.H., Swierstra, E.E. and Hunt, W.L. (1972). Spermatogenesis in the dog. Anat. Rec. 173: 341-352.

Freeman, C. and Coffey, D.S. (1973). Sterility in male animals induced by injection of chemical agents into the vas deferens. Fertil. Steril, 24: 884-890.

Immegart, H.M. and Threfall, W.R. (2000). Evaluation of Intratesticular injection of gossypol for non-surgical sterilization of dogs.Am. J. Vet.Res, 61(5):544-549.

Knobel, D.L., Cleaveland, S., Coleman, P.G., Fèvre, E.M., Meltzer, M.I. and Miranda, M.E. (2005). Re-evaluating the burden of rabies in Africa and Asia. Bull WHO. 83:360-368.

Koger, L.M. (1978). Calcium chloride castration. Modern Veterinary Practice. 59: 119-121.

Min Wang, M.D., Corbett, B., Maas, B. (2013). Effect of intra-testicular injection of zinc gluconate neutralized by arginine on reproductive parameters and testosterone levels in male dogs. $5^{\text {th }}$ International symposium on nonsurgical contraceptive methods of pet population control, Portland, Oregon, Pacific Northwest.

Murty, T.A and Sastry, G.A. (1978). The effects of cadmium chloride injection on the histopathology of the testis and the prostate in Dog. I. Intratesticular 
procedure. Indian.Vet. J., 55 (1): 368371.

Pineda, M.H. and Dolley, M.P. 1984. Surgical and chemical vasectomy in the cat. Am.J.Vet.Res. 45(2): 291-300.

Pineda, M.H., Reimers, T.J. and Faulkner, L.C. (1977). Azoospermia in dogs induced by injection of sclerosing agents into the cauda of the epididymides. Am.J.Vet.Res., 38: 831838.

Singh, G. 2004. Effects of chlorohexidine gluconate on epididymis and spermatogenesis in cow bulls. M.V.Sc., Thesis CCSHAU, Hisar (Haryana), India.
Tepsumethanon, V., Wildy, H. and Hemachudha, T. 2005. Intratesticular injection of balanced Zinc solution for permanent sterilization of Dogs. J.Med.Assoc.Thai. 88(5): 686-689.

Vannucchi, C.; Angrimani, D.S.; Eyherabide, A.R.; Mazzei, C.P.; Lucio, C.F, Maiorka P.C.; Silva, L.C.;Nichi, M.;2015. Effects of intratesticular administration of zinc gluconate and dimethyl sulfoxide on clinical, endocrinological, and reproductive parameters in dogs. Theriogenology. 84(7): 1103-10.

\section{How to cite this article:}

Rajan Chaudhary, Arun Kumar Sharma, Umed Singh Mehra, Ravi Dutt, Pankaj Kumar and Rajendra Yadav. 2018. Histopathological Studies on Intra Epididymal Zinc Arginine Administration in Pre-Pubertal Dogs. Int.J.Curr.Microbiol.App.Sci. 7(05): 569-581.

doi: https://doi.org/10.20546/ijcmas.2018.705.071 